\title{
Setipiprant for Androgenetic Alopecia in Males: Results from a Randomized, Double-Blind, Placebo-Controlled Phase 2a Trial
}

\author{
Janet DuBois' \\ Suzanne Bruce 2 \\ Daniel Stewart ${ }^{3}$ \\ Steven Kempers ${ }^{4}$ \\ Christy Harutunian ${ }^{5}$ \\ Terry Boodhoo ${ }^{5}$ \\ Amy Weitzenfeld ${ }^{6}$ \\ Joan-En Chang-Lin ${ }^{5}$ \\ 'DermResearch, Inc., Austin, TX, USA; \\ ${ }^{2}$ Suzanne Bruce and Associates, PA, \\ Houston, TX, USA; ${ }^{3}$ Midwest Center for \\ Dermatology \& Cosmetic Surgery, \\ Clinton Township, MI, USA; ${ }^{4}$ Associated \\ Skin Care Specialists, Fridley, MN, USA; \\ ${ }^{5}$ Allergan Aesthetics, an AbbVie \\ Company, Irvine, CA, USA; ${ }^{6}$ Allergan \\ Aesthetics, an AbbVie Company, \\ Madison, NJ, USA
}

Purpose: To evaluate oral setipiprant versus placebo for scalp hair growth in men with androgenetic alopecia (AGA).

Patients and Methods: Males aged 18 to 49 years with AGA were enrolled in a double-blind, multicenter, 32-week, phase 2a trial; randomized to twice-daily (BID) $1000-\mathrm{mg}(2 \times 500 \mathrm{mg}$ for a total daily dose of $2000 \mathrm{mg})$ setipiprant tablets or placebo for 24 weeks; and assessed at weeks $4,8,16$, and 24, with a week 32 follow-up. The study initially included a finasteride 1-mg once-daily group, removed by protocol amendment. Changes from baseline to week 24 in target area hair count (TAHC) and blinded Subject Self-Assessment (SSA) of target area photographs were coprimary efficacy endpoints. Hair growth was also evaluated using blinded Investigator Global Assessment (IGA). Safety assessments included adverse events (AEs) and clinical laboratory tests. Analysis of covariance models were used to test statistical significance for TAHC, SSA, and IGA. Data were summarized without statistical analysis for finasteride.

Results: Randomized subjects ( $\mathrm{N}=169)$ included 74 placebo, 83 setipiprant, and 12 finasteride subjects; $117(69.2 \%)$ and $113(66.9 \%)$ subjects completed week 24 and 32 visits, respectively. Treatment groups had similar baseline characteristics. Neither coprimary efficacy endpoint was met. At week 24, TAHC and SSA findings indicated no hair growth improvements with setipiprant versus placebo. Setipiprant also did not improve hair growth versus placebo per the IGA. Treatment-related AEs, all mild or moderate in severity, occurred in $12.3 \%, 25.9 \%$, and $25.0 \%$ of the placebo, setipiprant, and finasteride groups, respectively. Two treatment-emergent serious AEs (TESAEs), cellulitis and multiple sclerosis, were reported in the placebo group, both unrelated to treatment. No TESAEs were reported with setipiprant or finasteride.

Conclusion: Setipiprant $1000 \mathrm{mg}$ BID was safe and well tolerated but did not demonstrate efficacy versus placebo for scalp hair growth in men with AGA.

Keywords: scalp, hair loss, hair growth and development, prostaglandin receptors

\section{Plain Language Summary}

Androgenetic alopecia (AGA) is a common form of hair loss. In men, this condition is also known as male-pattern baldness. It develops at the top of the head or front of the scalp and worsens with age. Common treatments include finasteride and minoxidil. These treatments usually do not result in full hair regrowth. Setipiprant is an investigational oral medication for hair growth that works at specific possible molecular targets in the hair follicles. This was the first clinical study of the effect of setipiprant on hair growth in
Correspondence: Joan-En Chang-Lin

Clinical Development, Allergan

Aesthetics, an AbbVie Company, 2525

Dupont Dr, Irvine, CA, 92612, USA

$\mathrm{Tel}+\mid$ 7|4-246-4680

Email joanen.lin@abbvie.com 
men with AGA. The study compared setipiprant with a placebo treatment and, for part of the study, finasteride, an AGA-targeted, 5- $\alpha$-reductase inhibitor. Although the finasteride group was later removed to increase enrollment in the other study groups, a subset of subjects still completed the study. Men received treatment for 24 weeks and had follow-up visits through 32 weeks. Coprimary endpoints included change from baseline to week 24 in the target area hair count and in the subject's self-assessment of change in hair growth. Setipiprant did not cause significant improvements in hair growth compared with placebo, but it was safe and well tolerated. Although differences between the finasteride group and the setipiprant and placebo groups were not evaluated statistically, the value of mean change in the coprimary endpoints was numerically greater in the finasteride group, as expected.

\section{Introduction}

Androgenetic alopecia (AGA) in men, also known as male-pattern baldness, is hair loss at the top of the head (vertex) or front of the scalp that progresses with age. ${ }^{1}$ Factors leading to AGA may include alterations in the natural proportion of terminal hairs (thick and pigmented) to vellus hairs (short, fine, and nonpigmented), hair follicle miniaturization, and disruption in hair growth-cycle dynamics. $^{1,2}$ Individuals with AGA may experience sunburn or injury on the exposed scalp region, as well as psychosocial effects, including dissatisfaction with body image and the potential to be perceived as less attractive to others. $^{1,3}$

Common AGA treatments include finasteride (an oral 5 - $\alpha$-reductase type II inhibitor), minoxidil (a topical pyrimidine derivative), and hair transplantation. ${ }^{2,4-6}$ Hair transplantation may be costly and require multiple sessions. ${ }^{7}$ Finasteride and minoxidil slow hair loss, but neither results in full hair regrowth. ${ }^{2,5}$ Even with longterm treatment, the response to finasteride or minoxidil can be variable, and approximately $35 \%$ to $53 \%$ of men fail to show improvement in hair growth. ${ }^{8-10}$ In addition, finasteride may cause sexual side effects, such as erectile dysfunction, ejaculation dysfunction, and loss of libido. ${ }^{5}$ Alternative strategies that effectively prevent hair loss and target hair regrowth with novel mechanisms of action are being developed ${ }^{6}$ and would be valuable, especially for individuals for whom existing therapies have not been effective.

Testosterone has a known role in the pathophysiology of AGA; recently, the possibility of prostaglandin involvement has emerged, with different prostaglandins potentially possessing opposing functions. ${ }^{1,11}$ For example, the prostaglandin and prostaglandin-ethanolamide analogs latanoprost and bimatoprost, respectively, stimulate hair growth in vitro (eg, human tissue explants) and in vivo (eg, mouse and humans). ${ }^{12-15}$ In contrast, prostaglandin $\mathrm{D}_{2}\left(\mathrm{PGD}_{2}\right)$, a known proinflammatory mediator, ${ }^{16}$ and its receptor, chemoattractant receptor-homologous molecule expressed on Th2 cells (CRTH2; also known as G-protein-coupled receptor 44 [GPR44] or $\mathrm{PGD}_{2}$ receptor$2\left[\mathrm{DP}_{2}\right]$ ), inhibit hair lengthening ${ }^{17}$ and follicle regeneration after wounding ${ }^{18}$ in vitro (eg, human tissue explants) and in vivo (mouse models).

Setipiprant, an oral tetrahydropyridoindole derivative and selective CRTH2 antagonist, was designed to block $\mathrm{PGD}_{2}$ mediated inflammation, and in vitro assays demonstrated that setipiprant blocked eosinophil activation. ${ }^{19-22}$ In early-phase development studies for allergic rhinitis (AR) and asthma, setipiprant reduced airway hypersensitivity. ${ }^{16,20,22}$ Despite early positive findings, the AR clinical program for setipiprant was discontinued because of lack of efficacy in the laterphase trial. ${ }^{20}$ Given the role of $\mathrm{PGD}_{2}$-CRTH2 signaling in hair loss, setipiprant was investigated for treatment of AGA.

The present study is the first well-controlled study to assess the effect of a CRTH2 antagonist, setipiprant, on scalp hair growth in adult males with AGA. The safety, tolerability, and efficacy of oral setipiprant relative to placebo were evaluated.

\section{Materials and Methods Subjects}

Male subjects were enrolled if they were generally healthy and aged 18 to 49 years (amended from an initial maximum age of 41 years), had AGA vertex pattern type of IIIv, IV, or $\mathrm{V}$ on the Norwood-Hamilton Hair Loss Scale (NHS), ${ }^{23}$ and had dissatisfaction with overall hair appearance as measured by the Hair Satisfaction Assessment (HSA) scale (ie, HSA item 3 score of 4 or 5). Key exclusion criteria included history or evidence of hair loss for reasons other than AGA; scarring of the scalp or any condition or disease of the scalp, hair, or hair shaft (eg, seborrheic dermatitis, tinea infections, psoriatic dermatitis) that would impact assessment of efficacy or safety; use of topical products impacting hair growth within 6 months of study (12 months for systemic products); hair weaves or dyes used within the last 6 months; history of sensitivity to any components of the study 
interventions or tattoo ink that may affect efficacy evaluation; and past participation in another setipiprant study.

\section{Study Design}

This phase 2a, randomized, double-blind, placebo-controlled (for setipiprant only), multicenter study (ClinicalTrials.gov identifier NCT02781311) was conducted from July 2016 to May 2018 at 18 sites in the United States. The study consisted of 24 weeks of double-blind treatment and 8 weeks of safety follow-up. Institutional review boards or individual ethics committees (Cleveland Clinic IRB, Cleveland, $\mathrm{OH}$; University of Minnesota Human Research Protection Program, Minneapolis, MN; Wake Forest University Health Sciences IRB, Winston-Salem, NC; and Quorum Review, Inc., Seattle, WA) approved the study protocol and amendments before study initiation. The study was conducted according to Good Clinical Practice guidelines and principles stated in the Declaration of Helsinki. All subjects provided written informed consent before study procedures commenced.

\section{Treatment}

Subjects were randomized on day 1 to double-blind, twicedaily (BID) treatment with either placebo or setipiprant tablets $1000 \mathrm{mg}(2 \times 500 \mathrm{mg}$ for a daily total dose of $2000 \mathrm{mg}$ ) for 24 weeks (Figure 1). There were 2 randomization schemes: most subjects were randomized 1:1 to setipiprant or placebo, and a smaller cohort, selected from 2 centers where pharmacokinetics (PK) and scalp biopsy assessments would be performed, was randomized 5:1 to receive setipiprant or placebo. A subset of subjects also received the active comparator, finasteride 1-mg tablet once daily (QD) (Figure 1), until this treatment arm was removed by a protocol amendment designed to simplify the protocol and increase study enrollment. Subjects assigned to this group prior to the protocol amendment continued study intervention until completion of the study.

Study treatment was dispensed to subjects at the day 1 , week 4 , week 8 , and week 16 study visits. Subjects selfadministered the treatment, with instructions to take it with water at 12-hour intervals and without food (ie, 1 hour before or 2 hours after eating). A follow-up visit occurred at week 32 (8 weeks posttreatment). Evaluating investigators were blinded to all study interventions. However, finasteride treatment was not blinded to subjects and site personnel because of its identifiable appearance.

\section{Assessments}

Subjects returned at weeks $4,8,16$, and 24 for efficacy and/or safety assessments, as well as at week 32 or an early exit visit (if applicable) for follow-up safety and efficacy evaluations and procedures. Efficacy was assessed by objective, imaging-based methods and subjective, investigator- and subject-reported measures.

\section{Coprimary Efficacy Endpoints}

Change from baseline to week 24 in the target area hair count (TAHC), a standardized quantification of the number

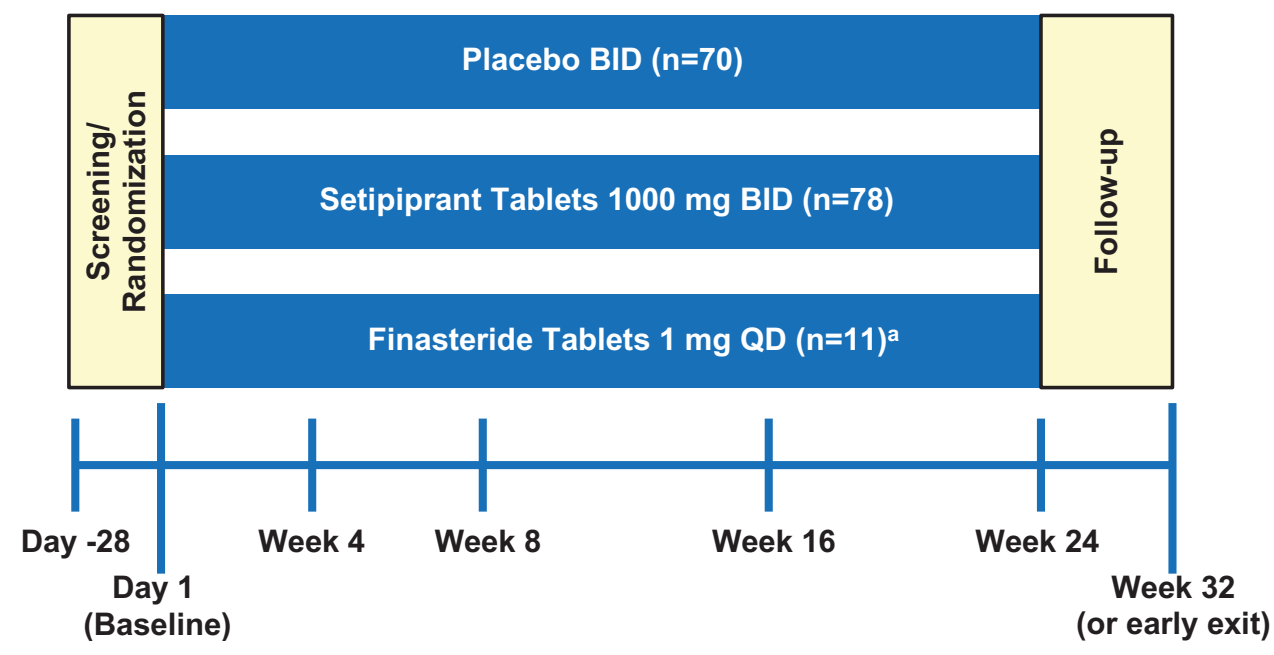

Figure I Study design (mITT population). The hair growth measures (TAHC, SSA, and IGA) were assessed at weeks 8, 16, 24, and 32 (or early study exit); adverse events were assessed at every study visit. Prespecified study visits are marked by blue vertical lines. ${ }^{\text {a }} \mathrm{A}$ once-daily finasteride I-mg arm was initially included in the study design but was removed to increase enrollment in the other arms.

Abbreviations: BID, twice daily; IGA, Investigator Global Assessment; mITT, modified intent to treat; QD, once daily; SSA, Subject Self-Assessment; TAHC, target area hair count. 
of terminal hairs ( $\geq 30 \mu \mathrm{m}$ wide), was a coprimary efficacy endpoint. TAHC was measured for each subject within a defined scalp region: the $1-\mathrm{cm}^{2}$ circular target area on the left side of the anterior leading edge of the thinning vertex area on subjects' scalps. Macrophotographs, centered on a semipermanent dot tattoo to ensure the same target area was characterized at each visit, were taken at baseline, at weeks 8,16 , and 24 during treatment, and at week 32 (follow-up) and analyzed with digital image analysis to assess TAHC.

The other coprimary endpoint was change from baseline to week 24 in blinded Subject Self-Assessment (SSA), the subject's own assessment of the change in hair growth, scored on a 7-point scale ranging from -3 (greatly decreased) to 3 (greatly increased). The higher the SSA score, the more perception of hair growth from baseline. Subjects assessed hair growth changes from paired global photographs showing before and after images of the vertex target area, which were presented to them in a blinded fashion.

\section{Other Efficacy Assessments}

Also evaluated was the change from baseline to week 24 in blinded Investigator Global Assessment (IGA), an investigators' assessment of the change in scalp hair growth scored on a 7-point scale ranging from -3 (greatly decreased) to 3 (greatly increased). The higher the global assessment score, the greater the perception of hair growth from baseline. Investigators assessed hair growth changes from paired global photographs showing before and after images of the subjects' vertex target area in a blinded fashion.

Blood samples for PK and genomic (CRTH2 polymorphisms) assessments were also collected. Pharmacokinetics were evaluated in plasma and scalp biopsies at 2 study centers, and genetic testing occurred at all centers (data on file; Allergan Aesthetics, an AbbVie Company).

\section{Safety}

Safety assessments included measures of treatment exposure, adverse event (AE) monitoring, vital sign measurements, clinical laboratory testing, electrocardiograms (ECGs), physical examination, and the occurrence of nonscalp hair growth. All laboratory results were reviewed, including any potential Hy's Law cases, defined as follows: alanine aminotransferase (ALT) and/or aspartate aminotransferase (AST) $\geq 3 \times$ the upper limit of normal (ULN), total bilirubin $\geq 2 \times$ $\mathrm{ULN}$, and alkaline phosphatase $<2 \times \mathrm{ULN},{ }^{24}$ all based on blood draws collected within a 24-hour period and no other explainable reason for the elevations.

\section{Statistical Analysis}

Least squares (LS) mean changes from baseline, LS mean differences between setipiprant and placebo, and $P$ values at each time point for the TAHC, SSA, and IGA endpoints were assessed using analysis of covariance (ANCOVA) models, with treatment as a fixed effect and age as a covariate. The ANCOVA model for TAHC included baseline values as a covariate. The type III sum of squares was used for all ANCOVA models. Two-sided $t$-tests were used to determine statistical significance, set at $P \leq 0.05$. For the coprimary endpoints (TAHC and SSA), missing data were imputed up to week 24 using the lastobservation-carried-forward approach. Missing data were not imputed for the IGA analysis because it was not a coprimary endpoint.

The number of subjects targeted for enrollment was approximately 152 , allowing for 80 and 72 subjects to enroll in the setipiprant and placebo arms, respectively. Assuming a $15 \%$ dropout rate and hair count treatment effects based on published estimates with finasteride, ${ }^{25,26}$ the target enrollment number would provide $92 \%$ power to detect treatment differences between groups.

The modified intent-to-treat (mITT) population included all randomized subjects who had 1 baseline and $\geq 1$ postbaseline measurement for 1 of the coprimary efficacy endpoints. The safety population comprised all subjects who received $\geq 1$ dose of study intervention. Data on subjects treated with finasteride were summarized alone (without applying the statistical analysis models).

\section{Results}

\section{Subject Disposition}

The number of randomized subjects totaled 169: 74 to placebo, 83 to setipiprant, and 12 to finasteride (Table 1). Of these, $117(69.2 \%)$ completed the primary endpoint visit at week 24 (ie, end of dosing). A total of 113 (66.9\%) of 169 randomized subjects completed the study through week 32. The reasons for discontinuation before week 32 in 56 subjects (33.1\%) are presented in Table 1.

\section{Demographics and Baseline Characteristics}

Demographics and baseline characteristics were generally similar between treatment arms (Table 2). Subjects in the finasteride group were somewhat younger compared with the other 2 groups because of the initial age maximum of 41 years (all finasteride-treated subjects were enrolled 
Table I Subject Disposition

\begin{tabular}{|l|c|c|c|}
\hline $\mathbf{n}$ (\%) & $\begin{array}{c}\text { Placebo } \\
\mathbf{( n = 7 4 )}\end{array}$ & $\begin{array}{c}\text { Setipiprant } \\
\mathbf{( n = 8 3 )}\end{array}$ & $\begin{array}{c}\text { Finasteride } \\
\text { (n=12) }\end{array}$ \\
\hline mITT population & $70(94.6)$ & $78(94.0)$ & $11(91.7)$ \\
\hline Safety population & $73(98.6)$ & $81(97.6)$ & $12(100)$ \\
\hline Completed study & $48(64.9)$ & $57(68.7)$ & $8(66.7)$ \\
\hline Prematurely discontinued study & $26(35.1)$ & $26(31.3)$ & $4(33.3)$ \\
Withdrawal of consent & $11(14.9)$ & $12(14.5)$ & $2(16.7)$ \\
Lost to follow-up & $11(14.9)$ & $8(9.6)$ & $1(8.3)$ \\
Adverse event & $2(2.7)$ & $6(7.2)$ & $1(8.3)$ \\
Noncompliance with study drug & $2(2.7)$ & 0 & 0 \\
\hline
\end{tabular}

Note: ${ }^{\text {a}}$ There were no discontinuations due to lack of efficacy or protocol violations.

Abbreviation: mITT, modified intent-to-treat.

Table 2 Demographics and Baseline Characteristics (mITT Population)

\begin{tabular}{|c|c|c|c|}
\hline Characteristic & $\begin{array}{l}\text { Placebo } \\
(n=70)\end{array}$ & $\begin{array}{c}\text { Setipiprant } \\
\quad(n=78)\end{array}$ & $\begin{array}{c}\text { Finasteride } \\
\qquad(n=I I)\end{array}$ \\
\hline Mean (SD) age, $y$ & $36.9(6.1)$ & $36.3(6.7)$ & $34.1(3.1)$ \\
\hline \multicolumn{4}{|l|}{ Race, n (\%) } \\
\hline White & $59(84.3)$ & $70(89.7)$ & $9(81.8)$ \\
\hline Black or African American & $4(5.7)$ & $3(3.8)$ & 0 \\
\hline Asian & $4(5.7)$ & $\mathrm{I}(\mathrm{I} .3)$ & I (9.1) \\
\hline Other ${ }^{a}$ & $3(4.3)$ & $3(3.8)^{\mathrm{b}}$ & I (9.1) \\
\hline \multicolumn{4}{|l|}{ NHS, n (\%) } \\
\hline III Vertex & $22(3 \mathrm{I} .4)$ & $26(33.3)$ & $6(54.5)$ \\
\hline IV & I8 (25.7) & $20(25.6)$ & 0 \\
\hline$\vee$ & $30(42.9)$ & $32(4 I .0)$ & $5(45.5)$ \\
\hline \multicolumn{4}{|c|}{ Mean (SD) TAHC, terminal hairs $/ \mathrm{cm}^{2}$} \\
\hline Left side & I $36.7(55.8)$ & I $48.6(64.6)$ & I $39.9(47.7)$ \\
\hline
\end{tabular}

Notes: ${ }^{a}$ Other comprised American Indians or Alaska natives, native Hawaiians or other Pacific Islanders, and subjects who reported $\geq 2$ races. ${ }^{b}$ One subject treated with setipiprant had missing data for race and was not included in this count.

Abbreviations: mITT, modified intent-to-treat; NHS, Norwood-Hamilton Scale; TAHC, target area hair count.

under this inclusion criterion). Most subjects (75.5\%) had experienced hair loss for 5 or more years.

\section{Efficacy}

\section{Coprimary Endpoints}

Differences between setipiprant and placebo were not statistically significant at week 24 for either coprimary endpoint. At week 24, TAHC increased from baseline in all treatment groups (Figure 2). The mean change from baseline in TAHC in the placebo, setipiprant, and finasteride groups, respectively, was 7.0, 6.7, and 33.9 terminal hairs/ $\mathrm{cm}^{2}$. The LS mean difference between setipiprant and placebo at week 24 was not statistically significant
( $P=0.92$; Table 3 ), with similar findings observed at the other time points.

Similarly, subjects did not indicate improvements in hair growth with either placebo or setipiprant at week 24 using the SSA (Figure 3). The mean change from baseline in SSA in the placebo, setipiprant, and finasteride groups was $-0.2,-0.3$, and 0.8 , respectively. The LS mean difference between setipiprant and placebo on the SSA was not statistically significant at week 24 ( $P=0.91$; Table 3 ) or other time points. As demonstrated in Figures 2 and 3, the mean changes from baseline in TAHC and SSA were greater in the finasteride group than in the setipiprant and placebo groups, but a formal statistical evaluation comparing finasteride and placebo was not conducted. 


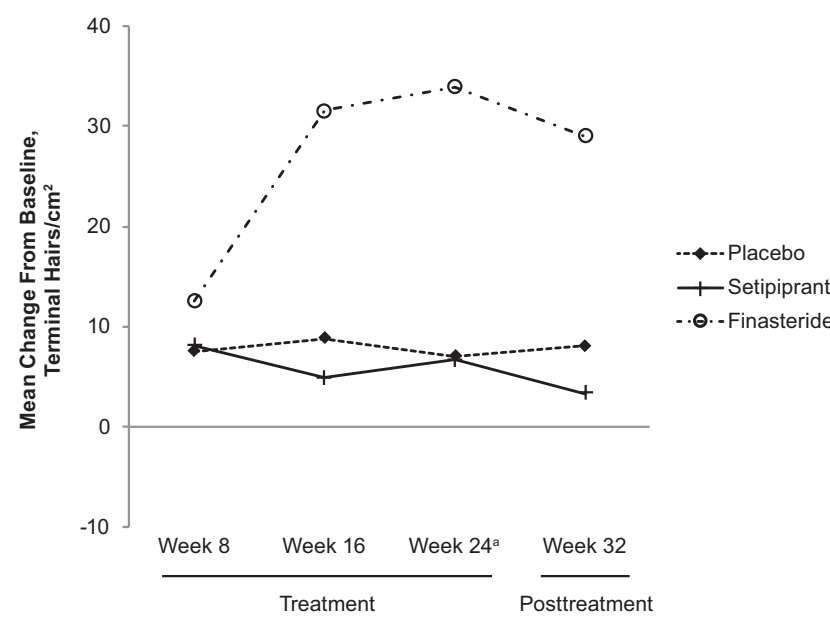

Figure 2 Target area hair count mean change from baseline by time point (mITT population). Data shown are within a $1-\mathrm{cm}^{2}$ circular area on the left side of the

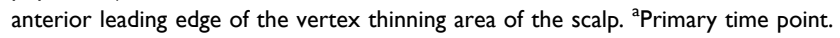
Abbreviation: mITT, modified intent-to-treat.

\section{Other Efficacy Endpoints}

Investigators did not detect improvements in hair growth in the placebo or setipiprant groups at week 24 using the IGA (Figure 4). Mean change in IGA in the placebo, setipiprant, and finasteride groups was $-0.3,-0.3$, and 0.2 , respectively. The difference between setipiprant and placebo for this endpoint was not statistically significant at week $24(P=0.85$; Table 3) or other time points. As with the other efficacy measures, treatment with finasteride resulted in improvement on the IGA from baseline to week 24 (Figure 4). Representative subject photographs before and after treatment (week 24) are shown in Figure 5.

\section{Safety}

The mean duration of exposure was 19 to 20 weeks for all treatment groups. The incidence of treatment-emergent AEs (TEAEs) was $43.8 \%, 54.3 \%$, and $58.3 \%$ in the placebo, setipiprant, and finasteride groups, respectively (Table 4). Investigators assessed most TEAEs as mild in severity. The most frequently reported TEAEs were sinusitis in the placebo group $(n=4 ; 5.5 \%)$; upper respiratory tract infection (URTI) and AST increased in the setipiprant group ( $\mathrm{n}=6$ each; 7.4\%); and URTI and libido decreased in the finasteride group ( $\mathrm{n}=2$ each; 16.7\%; Table 4).

Treatment-related TEAEs, all mild or moderate in severity, occurred in $12.3 \%, 25.9 \%$, and $25.0 \%$ of the placebo, setipiprant, and finasteride groups, respectively (Table 4). Those occurring in $\geq 2$ subjects in the setipiprant group were ALT increased and weight increased $(n=4$ each; 4.9\%), hair growth abnormal and AST increased $(\mathrm{n}=3$ each; 3.7\%), and abdominal pain upper and diarrhea $(\mathrm{n}=2$ each; $2.5 \%)$.

Two subjects $(2.7 \%)$ in the placebo group, 6 subjects (7.4\%) in the setipiprant group, and 1 subject (8.3\%) in the finasteride group discontinued the study due to TEAEs (Table 4). Two serious TEAEs (multiple sclerosis and cellulitis) were reported in the study; both were in the placebo group and were considered by the investigator as unrelated to study treatment. There were no reported deaths during the study.

No meaningful differences in elevations of liver function tests between the setipiprant and placebo groups were

Table 3 Efficacy Endpoints at Week 24: LS Mean Change from Baseline and LS Mean Difference Between Setipiprant and Placebo Using ANCOVA (mITT Population)

\begin{tabular}{|c|c|c|}
\hline & Placebo & Setipiprant \\
\hline Target Area Hair Count, terminal hairs $/ \mathrm{cm}^{2}$ & $\mathrm{n}=6 \mathrm{l}$ & $n=70$ \\
\hline LS mean change from baseline (SE) & $6.7(3.3)$ & $7.1(3.0)$ \\
\hline LS mean difference $(95 \% \mathrm{Cl})^{\mathrm{a}}$ & \multicolumn{2}{|c|}{$0.4(-8.4$ to 9.2$)$} \\
\hline$P$ value & \multicolumn{2}{|c|}{0.92} \\
\hline Subject Self-Assessment & $n=68$ & $n=78$ \\
\hline LS mean change from baseline (SE) & $-0.2(0.2)$ & $-0.3(0.1)$ \\
\hline LS mean difference $(95 \% \mathrm{Cl})^{\mathrm{a}}$ & \multicolumn{2}{|c|}{$0.0(-0.4$ to 0.4$)$} \\
\hline$P$ value & \multicolumn{2}{|c|}{0.91} \\
\hline Investigator Global Assessment & $\mathrm{n}=48$ & $n=60$ \\
\hline LS mean change from baseline (SE) & $-0.3(0.2)$ & $-0.3(0.1)$ \\
\hline LS mean difference $(95 \% \mathrm{Cl})^{\mathrm{a}}$ & \multicolumn{2}{|c|}{$0.0(-0.4$ to 0.4$)$} \\
\hline$P$ value & \multicolumn{2}{|c|}{0.85} \\
\hline
\end{tabular}

Note: ${ }^{a}$ Setipiprant vs placebo.

Abbreviations: ANCOVA, analysis of covariance; LS, least squares; mITT, modified intent-to-treat. 


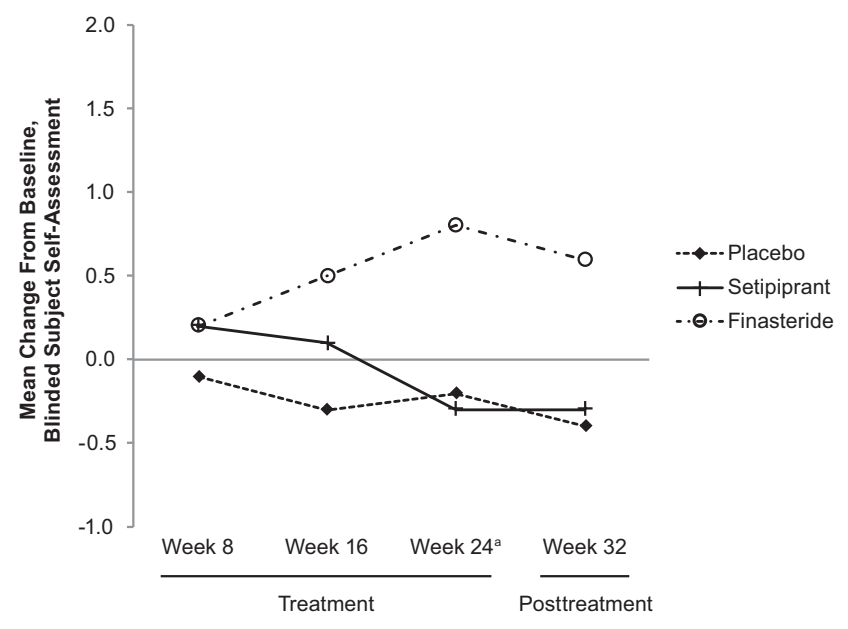

Figure 3 Subject Self-Assessment (SSA) of hair growth mean change from baseline

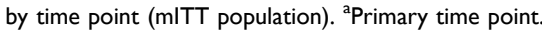

Abbreviation: mITT, modified intent-to-treat.

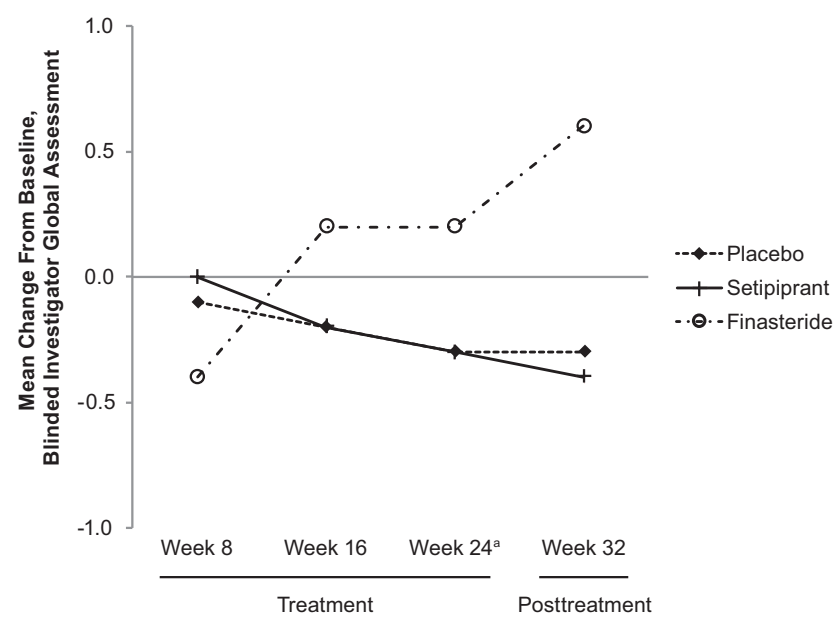

Figure 4 Investigator Global Assessment (IGA) of hair growth mean change from baseline by time point (mITT population). ${ }^{\text {a }}$ Primary time point.

Abbreviation: mITT, modified intent-to-treat.

found (Table 5). None of the subjects with aminotransferase elevations ( $\geq 3 \times \mathrm{ULN}$ ) had elevated total bilirubin ( $\geq 2 \times$ ULN), and thus none met the laboratory criteria for a potential case of Hy's Law (indicative of possible druginduced liver injury). ${ }^{24}$

\section{Discussion}

This randomized, placebo-controlled, double-blind trial was the first to evaluate an oral CRTH2 antagonist, setipiprant, versus placebo on scalp hair growth in men with AGA. Neither coprimary efficacy endpoint was met: at week 24, there were increases in TAHC across all treatment groups, but between-group differences were not statistically significant. SSA findings indicated no improvements in hair growth with setipiprant. Additionally, setipiprant did not improve hair growth versus placebo per the IGA.

Although a protocol amendment removed the finasteride treatment arm from this study, a small cohort of subjects was treated in an unblinded fashion until week 24 . The differences between the finasteride group and the setipiprant and placebo groups were not formally evaluated for statistical significance, but the mean improvements in the coprimary endpoints were numerically greater with finasteride. Outcomes with finasteride were as expected based on prior data. ${ }^{27}$

Overall, setipiprant was safe and well tolerated. Most TEAEs were mild and no serious TEAEs were reported in the setipiprant group. Although the safety of setipiprant for men with AGA was shown, efficacy in this population was not demonstrated. Lack of drug exposure at the target area after oral dosing is unlikely to account for the lack of efficacy in this study, given that preclinical models ${ }^{19}$ demonstrated high levels of oral uptake and exposure. Furthermore, in the present study, the PK analysis in plasma samples confirmed exposure as expected (data on file, Allergan Aesthetics, an AbbVie Company). ${ }^{20,22,28}$ PK data will be described in further detail in a separate publication. Scalp biopsy samples also exhibited drug concentrations greater than or equal to those estimated to target CRTH2 or to block $\mathrm{PGD}_{2}$-mediated inhibition of hair growth (data on file, Allergan Aesthetics, an AbbVie Company). These findings appear consistent with those of previous clinical studies with setipiprant for AR and asthma, in which early results showed an effect, but later studies in AR demonstrated a lack of efficacy despite adequate drug levels. ${ }^{20,22}$ Either the theoretical role of $\mathrm{PGD}_{2}$-CRTH2 signaling in hair loss does not directly translate to clinical outcomes in AGA, or even higher doses may be required. Depending on the exact concentrations of setipiprant found in the scalp after oral dosing (ie, whether it is in the low or high end of the target concentration to elicit a pharmacologic response), it may also be worth considering whether delivering the drug directly to the target site via topical administration yields higher drug concentrations compared with oral dosing, especially if there are limitations to using oral doses that are higher than what is reported here. Successful topical delivery will depend on the system used to deliver therapeutic levels of setipiprant across the dermal barrier to the target site.

The small sample size of this study may limit the interpretation of these findings. However, consistent outcomes across multiple efficacy endpoints strengthen the 

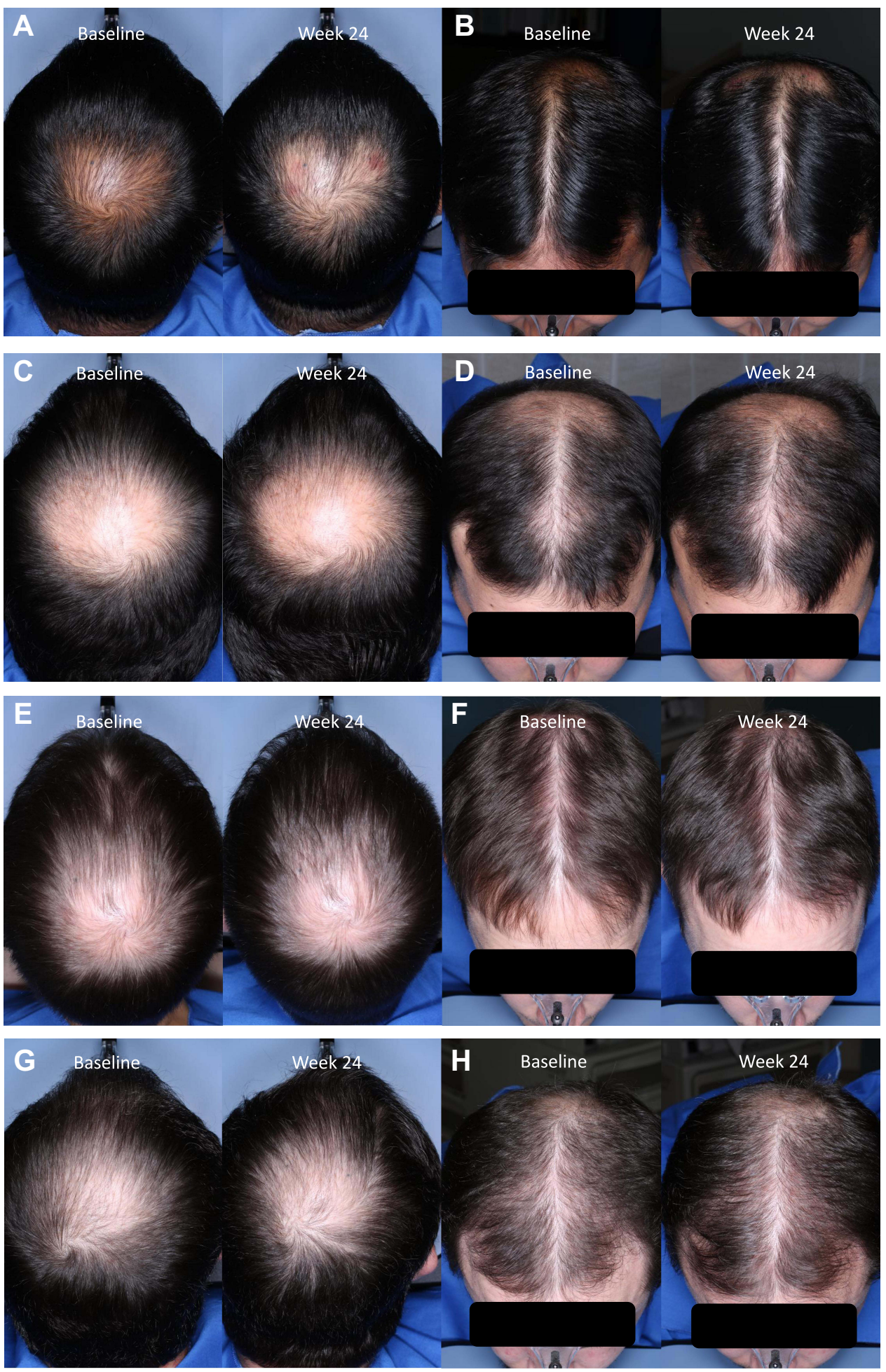

Figure 5 Representative pre- (baseline) and posttreatment (week 24) photographs of subjects with androgenetic alopecia treated twice daily with setipiprant tablets $1000 \mathrm{mg}(2 \times 500 \mathrm{mg}$ for a daily total dose of $2000 \mathrm{mg})$ for 24 weeks. Photographs show the vertex and frontal/superior views of a $4 \mathrm{I}$-year-old subject (A and B), a 32 -yearold subject (C and $\mathbf{D})$, a 39-year-old subject (E and $\mathbf{F})$, and a 37-year-old subject ( $\mathbf{G}$ and $\mathbf{H})$.

confidence in the overall conclusions. This study assessed the effects of setipiprant on hair loss only in men with AGA and not in women with female-pattern hair loss.
However, differences in response according to the sex of the subject were not expected based on the mechanism of action of setipiprant or the outcomes assessed. 
Table 4 Adverse Events (Safety Population)

\begin{tabular}{|c|c|c|c|}
\hline Subjects, n (\%) & $\begin{array}{c}\text { Placebo } \\
(n=73)\end{array}$ & $\begin{array}{c}\text { Setipiprant } \\
\quad(n=8 I)\end{array}$ & $\begin{array}{l}\text { Finasteride } \\
\qquad(n=12)\end{array}$ \\
\hline TEAEs & $32(43.8)$ & $44(54.3)$ & $7(58.3)$ \\
\hline Treatment-related TEAEs & $9(12.3)$ & $21(25.9)$ & $3(25.0)$ \\
\hline Treatment-emergent SAEs & $2(2.7)$ & 0 & 0 \\
\hline TEAEs leading to discontinuation ${ }^{a}$ & $2(2.7)$ & $6(7.4)$ & I (8.3) \\
\hline \multicolumn{4}{|l|}{ TEAEs occurring in $\geq 5 \%$ in any group } \\
\hline Agitation & 0 & 0 & I (8.3) \\
\hline Arthralgia & 0 & 0 & I (8.3) \\
\hline AST increased & $2(2.7)$ & $6(7.4)$ & I (8.3) \\
\hline Diarrhea & $2(2.7)$ & $4(4.9)$ & I (8.3) \\
\hline Dyspnea & 0 & 0 & I (8.3) \\
\hline Fatigue & $\mathrm{I}(\mathrm{l} .4)$ & 0 & I (8.3) \\
\hline Libido decreased & 0 & 0 & $2(16.7)$ \\
\hline Oropharyngeal pain & 0 & 0 & $\mathrm{I}(8.3)$ \\
\hline Pruritus & 0 & 0 & I (8.3) \\
\hline Rash generalized & 0 & 0 & I (8.3) \\
\hline Sinusitis & $4(5.5)$ & $3(3.7)$ & 0 \\
\hline Skin abrasion & 0 & $\mathrm{I}(\mathrm{I} .2)$ & I (8.3) \\
\hline Swelling face & 0 & 0 & I (8.3) \\
\hline Upper respiratory tract infection & 0 & $6(7.4)$ & $2(16.7)$ \\
\hline Vomiting & 0 & $\mathrm{I}(1.2)$ & I (8.3) \\
\hline
\end{tabular}

Note: ${ }^{a}$ TEAEs leading to discontinuation were multiple sclerosis and testicular pain in the placebo group; abdominal pain, alanine aminotransferase (ALT) increased ( 2 events), AST increased, diarrhea, gastroenteritis, headache, and pleurisy in the setipiprant group; and fatigue and libido decreased in the finasteride group.

Abbreviations: AST, aspartate aminotransferase; SAE, serious adverse event; TEAE, treatment-emergent adverse event.

Table 5 PCS Postbaseline Liver Function Tests in Subjects with Non-PCS Baseline Values (Safety Population)

\begin{tabular}{|l|c|c|c|}
\hline & $\begin{array}{c}\text { Placebo } \\
(\mathbf{n}=\mathbf{7 3})\end{array}$ & $\begin{array}{c}\text { Setipiprant } \\
(\mathbf{n}=\mathbf{8} \mathbf{I})\end{array}$ & $\begin{array}{c}\text { Finasteride } \\
(\mathbf{n}=\mathbf{1} \mathbf{2})\end{array}$ \\
\hline ALT $\geq 3 \times$ ULN & $3(4.1)$ & $4(4.9)$ & 0 \\
AST $\geq 3 \times$ ULN & $3(4.1)$ & $3(3.7)$ & $1(8.3)$ \\
ALT and/or AST $\geq 3 \times$ ULN & $5(6.9)$ & $5(6.2)$ & $1(8.3)$ \\
\hline
\end{tabular}

Abbreviations: ALT, alanine aminotransferase; AST, aspartate aminotransferase; PCS, potentially clinically significant; ULN, upper limit of normal.

\section{Conclusions}

In this population of adult males with AGA, setipiprant $1000 \mathrm{mg}$ BID was generally safe and well tolerated but did not demonstrate statistically significant efficacy differences from placebo. There were no clinically significant changes in safety parameters, and no safety signals were identified. This is the first clinical study testing setipiprant targeting the potential molecular mechanisms underlying hair growth in AGA.

\section{Abbreviations}

AE, adverse event; AGA, androgenetic alopecia; ALT, alanine aminotransferase; ANCOVA, analysis of covariance; AR, allergic rhinitis; AST, aspartate aminotransferase; BID, twice daily; CRTH2, chemoattractant receptor-homologous molecule expressed on Th2 cells; $\mathrm{DP}_{2}, \mathrm{PGD}_{2}$ receptor-2; ECG, electrocardiogram; GPR44, G-protein-coupled receptor 44; HSA, Hair Satisfaction Assessment; IGA, Investigator Global Assessment; LS, least squares; mITT, modified intentto-treat; NHS, Norwood-Hamilton Hair Loss Scale; PCS, potentially clinically significant; $\mathrm{PGD}_{2}$, prostaglandin $\mathrm{D}_{2}$; PK, pharmacokinetics; QD, once daily; SAE, serious adverse event; SSA, Subject Self-Assessment; TAHC, target area hair count; TEAE, treatment-emergent adverse event; ULN, upper limit of normal; URTI, upper respiratory tract infection.

\section{Data Sharing Statement}

AbbVie is committed to responsible data sharing regarding the clinical trials we sponsor. This includes access to 
anonymized, individual and trial-level data (analysis data sets), as well as other information (eg, protocols and Clinical Study Reports), as long as the trials are not part of an ongoing or planned regulatory submission. This includes requests for clinical trial data for unlicensed products and indications.

This clinical trial data can be requested by any qualified researchers who engage in rigorous, independent scientific research, and will be provided following review and approval of a research proposal and Statistical Analysis Plan (SAP) and execution of a Data Sharing Agreement (DSA). Data requests can be submitted at any time and the data will be accessible for 12 months, with possible extensions considered. For more information on the process, or to submit a request, visit the following link: https://www.abbvie.com/our-science/clinicaltrials/clinical-trials-data-and-information-sharing/data-andinformation-sharing-with-qualified-researchers.html.

\section{Acknowledgments}

All authors meet the ICMJE authorship criteria. The authors thank the participating patients, investigators, and study sites; former Allergan employee Gurpreet Ahluwalia for his initial clinical study design and clinical development expertise; Paige Pearson from Allergan data management; and Susan $\mathrm{Na}$ and Suzanne MacKinnon from Allergan clinical operations.

\section{Funding}

This study was sponsored by Allergan Aesthetics, an AbbVie Company, Dublin, Ireland. Writing and editorial assistance was provided to the authors by Regina Kelly, MA, of Peloton Advantage, LLC, an OPEN Health company, Parsippany, NJ, USA, and was funded by Allergan Aesthetics, an AbbVie Company. Neither honoraria nor other forms of payment were made for authorship.

\section{Disclosures}

Terry Boodhoo is an employee of Allergan Aesthetics, an AbbVie Company, and may hold AbbVie stock. Suzanne Bruce has received funding from Allergan-related participation in advisory boards and clinical trial conduct and training; and clinical trial funding for alopecia trials from Concert Pharmaceuticals, Aclaris Therapeutics, Incyte Corporation, and Eli Lilly and Company. She also reports personal fees from AbbVie, advisory board for Almirall, grants from Amgen, Arcutis, Biofrontera AG, Brickell Biotech, Chemocentryx, Dermavant, ENDO Pharmaceuticals,
Galderma, Pfizer, and Pulse BioScience, outside the submitted work. Amy Weitzenfeld is an employee of Allergan Aesthetics, an AbbVie Company, and may hold AbbVie stock. Janet DuBois has received clinical trial funding for androgenetic alopecia trials from Allergan Aesthetics, an AbbVie Company, and Aclaris Therapeutics. She also reports grants for clinical trials from Arcutis, Inc., Atacama Therapeutics, Bellus Health, Biofrontera, Boston Pharmaceuticals, Brickell Biotech, Inc., Bristol-Myers Squibb, Cara Therapeutics, Dermata Therapeutics, Endo International plc, Galderma, Incyte, Leo Pharma, NFlection Therapeutics, Inc., RAPT Therapeutics, Almirall, AiViva Biopharma, Inc., Bausch Health Americas, Merck Sharp \& Dohme Corp., and Palvella Therapeutics, Inc. outside the submitted work. Christy Harutunian is an employee of Allergan Aesthetics, an AbbVie Company, and may hold AbbVie stock. Steven Kempers is a consultant and clinical trial investigator for Arcutis Biotherapeutics, as well as a clinical trial investigator for Concert Pharmaceuticals, Incyte Corporation, Eli Lilly and Company, AbbVie, Bristol Myers Squibb, Forte Biosciences, Timber Pharmaceuticals, Dermira, Amgen, Galderma, Brickell Biotech, Edesa Biotech, Candesant Biomedical, Novan, NFlection Therapeutics, Novartis, and Palvella Therapeutics. Joan-En Chang-Lin is an employee of Allergan Aesthetics, an AbbVie Company, and may hold AbbVie stock. Daniel Stewart owns AbbVie stock. The authors report no other conflicts of interest in this work.

\section{References}

1. Stough D, Stenn K, Haber R, et al. Psychological effect, pathophysiology, and management of androgenetic alopecia in men. Mayo Clin Proc. 2005;80(10):1316-1322. doi:10.4065/80.10.1316

2. Rathnayake D, Sinclair R. Male androgenetic alopecia. Expert Opin Pharmacother. 2010;11(8):1295-1304. doi:10.1517/14656561003752730

3. Cash TF. The psychological effects of androgenetic alopecia in men. $J \mathrm{Am}$ Acad Dermatol. 1992;26(6):926-931. doi:10.1016/0190-9622(92)70134-2

4. Kanti V, Messenger A, Dobos G, et al. Evidence-based (S3) guideline for the treatment of androgenetic alopecia in women and in men - short version. $J$ Eur Acad Dermatol Venereol. 2018;32(1):11-22. doi: $10.1111 /$ jdv. 14624

5. Kanti V, Messenger A, Dobos G, et al. European Dermatology Forum Guideline for the Treatment of Androgenetic Alopecia in Women and in Men. Zurich, Switzerland: European Dermatology Forum; 2017.

6. Rossi A, Anzalone A, Fortuna MC, et al. Multi-therapies in androgenetic alopecia: review and clinical experiences. Dermatol Ther. 2016;29(6):424-432. doi:10.1111/dth.12390

7. Almohanna HM, Perper M, Tosti A. Safety concerns when using novel medications to treat alopecia. Expert Opin Drug Saf. 2018;17 (11):1115-1128. doi:10.1080/14740338.2018.1533549

8. Roberts JL, Fiedler V, Imperato-McGinley J, et al. Clinical dose ranging studies with finasteride, a type 2 5alpha-reductase inhibitor, in men with male pattern hair loss. J Am Acad Dermatol. 1999;41 (4):555-563. 
9. Kaufman KD, Dawber RP. Finasteride, a type 2 5alpha-reductase inhibitor, in the treatment of men with androgenetic alopecia. Expert Opin Investig Drugs. 1999;8(4):403-415. doi:10.1517/13543784.8.4.403

10. Olsen EA, Dunlap FE, Funicella T, et al. A randomized clinical trial of $5 \%$ topical minoxidil versus $2 \%$ topical minoxidil and placebo in the treatment of androgenetic alopecia in men. $J$ Am Acad Dermatol. 2002;47(3):377-385. doi:10.1067/mjd.2002.124088

11. Nieves A, Garza LA. Does prostaglandin D2 hold the cure to male pattern baldness? Exp Dermatol. 2014;23(4):224-227. doi:10.1111/ exd. 12348

12. Woodward DF, Liang Y, Krauss AH. Prostamides (prostaglandin-ethanolamides) and their pharmacology. $\mathrm{Br}$ J Pharmacol. 2008;153(3):410-419. doi:10.1038/sj.bjp.0707434

13. Khidhir KG, Woodward DF, Farjo NP, et al. The prostamide-related glaucoma therapy, bimatoprost, offers a novel approach for treating scalp alopecias. FASEB J. 2013;27(2):557-567. doi:10.1096/fj.12218156

14. Johnstone MA, Albert DM. Prostaglandin-induced hair growth. Surv Ophthalmol. 2002;47(Suppl 1):S185-S202. doi:10.1016/S00396257(02)00307-7

15. Gandolfi S, Simmons ST, Sturm R, Chen K, VanDenburgh AM. Three-month comparison of bimatoprost and latanoprost in patients with glaucoma and ocular hypertension. Adv Ther. 2001;18 (3):110-121. doi:10.1007/BF02850299

16. Kupczyk M, Kuna P. Targeting the PGD2/CRTH2/DP1 signaling pathway in asthma and allergic disease: current status and future perspectives. Drugs. 2017;77(12):1281-1294. doi:10.1007/s40265017-0777-2

17. Garza LA, Liu Y, Yang Z, et al. Prostaglandin D2 inhibits hair growth and is elevated in bald scalp of men with androgenetic alopecia. $\mathrm{Sci}$ Transl Med. 2012;4(126):126ra134. doi:10.1126/scitranslmed.3 003122

18. Nelson AM, Loy DE, Lawson JA, Katseff AS, Fitzgerald GA, Garza LA. Prostaglandin D2 inhibits wound-induced hair follicle neogenesis through the receptor, Gpr44. J Invest Dermatol. 2013;133(4):881-889. doi:10.1038/jid.2012.398

19. Fretz H, Valdenaire A, Pothier J, et al. Identification of 2-(2-(1-naphthoyl)-8-fluoro-3,4-dihydro-1H-pyrido[4,3-b]indol-5 (2H)-yl)acetic acid (setipiprant/ACT-129968), a potent, selective, and orally bioavailable chemoattractant receptor-homologous molecule expressed on Th2 cells (CRTH2) antagonist. J Med Chem. 2013;56 (12):4899-4911.
20. Ratner P, Andrews CP, Hampel FC, et al. Efficacy and safety of setipiprant in seasonal allergic rhinitis: results from Phase 2 and Phase 3 randomized, double-blind, placebo- and active-referenced studies. Allergy Asthma Clin Immunol. 2017;13:18. doi:10.1186/ s13223-017-0183-z

21. Willetts L, Ochkur SI, Jacobsen EA, Lee JJ, Lacy P. Eosinophil shape change and secretion. Methods Mol Biol. 2014;1178:111-128.

22. Diamant Z, Sidharta PN, Singh D, et al. Setipiprant, a selective CRTH2 antagonist, reduces allergen-induced airway responses in allergic asthmatics. Clin Exp Allergy. 2014;44(8):1044-1052. doi:10.1111/cea.12357

23. Norwood OT. Male pattern baldness: classification and incidence. South Med J. 1975;68(11):1359-1365. doi:10.1097/00007611197511000-00009

24. Guidance for industry: drug-induced liver injury: premarketing clinical evaluation; 2009. Available from: https://www.fda.gov/media/ 116737/download. Accessed September 30, 2021.

25. Gubelin Harcha W, Barboza Martinez J, Tsai TF, et al. A randomized, active- and placebo-controlled study of the efficacy and safety of different doses of dutasteride versus placebo and finasteride in the treatment of male subjects with androgenetic alopecia. $\mathrm{J} \mathrm{Am} \mathrm{Acad}$ Dermatol. 2014;70(3):489-498.e483. doi:10.1016/j.jaad.2013.10.049

26. Olsen EA, Hordinsky M, Whiting D, et al. The importance of dual $5 \alpha$-reductase inhibition in the treatment of male pattern hair loss: results of a randomized placebo-controlled study of dutasteride versus finasteride. J Am Acad Dermatol. 2006;55(6):1014-1023. doi:10.1016/j.jaad.2006.05.007

27. Mella JM, Perret MC, Manzotti M, Catalano HN, Guyatt G. Efficacy and safety of finasteride therapy for androgenetic alopecia: a systematic review. Arch Dermatol. 2010;146(10):1141-1150. doi:10.1001/archdermatol.2010.256

28. Sidharta PN, Diamant Z, Dingemanse J. Single- and multiple-dose tolerability and pharmacokinetics of the CRTH2 antagonist setipiprant in healthy male subjects. Fundam Clin Pharmacol. 2014;28 (6):690-699. doi:10.1111/fcp.12079
Clinical, Cosmetic and Investigational Dermatology is an international, peer-reviewed, open access, online journal that focuses on the latest clinical and experimental research in all aspects of skin disease and cosmetic interventions. This journal is indexed on CAS
The manuscript management system is completely online and includes a very quick and fair peer-review system, which is all easy to use. Visit http://www.dovepress.com/testimonials.php to read real quotes from published authors. 